\title{
Control System Modeling And Solving For The Multi-objective Level Balance
}

\author{
HUANG Jinlin ${ }^{1,}$, , ZHOU Keliang ${ }^{2, a}$ WANG Chen ${ }^{1, a}$ \\ ${ }^{1}$ Anhui Technological College of Machinery and Electricity, Wuhu 241000, China \\ ${ }^{2}$ School of Electrical Engineering and Automation, Jiangxi University of Science and Technology, \\ Ganzhou, China \\ ahuangjinlin0797@163.com
}

Keywords: Multi-objective, Mathematical Model, Level, Balance.

Abstract. During the production process of metal hydrometallurgical based on the Multi-objective Level Balance Control System, advantages such as ensure the production, improve production efficiency, reduce the cost can be made if the level can self-balancing. This paper around the problem of the level balance in six towers during the production process of metal hydrometallurgical, introduced the process. Equations are established according to the adsorption material in six tower's balanced relationship, we have to use material balance principle to establish the mathematical model of level's balance. According to the initial level of the six towers can be derived the amount of adsorption material flows to the next tower in each production cycle. Solved by Matlab, analysis results show that based on the least production cycle. This model can keep the six tower's level remain within the error range.This template explains and demonstrates how to prepare your camera-ready paper for Trans Tech Publications. The best is to read these instructions and follow the outline of this text.

\section{Introduction}

With the development of the production process, automatic control has been developed, calculation and production are connected indivisible. Calculation is not only the basic skill, but also an important part of automation equipment[1]. Using craft which is composed with oxygen and alkali has made the leaching rate increased greatly in America, has exploited the drill and construction craft[2]. In our country, hydrometallurgical factories focused on increased the overall rate of mineral resources by using advanced science and technology, exploited and utilized the limited resources fully and draw into the underground heaping technology, so the leaching rate has been some improvement, but for the study of the metal resource's feed, emission and enhance step, shortage still exists.

In order to ensure the product quality and reduce the cost, there are six towers in the Multi-objective Control System of the hydrometallurgical productive process. It requires each tower reaching the same high in the productive process. This system belongs to a Multi-objective Control System[3]. The tower is characteristic by coupling and internal circulation relationship. The limit of flux is the capacity of the scan which is an Multi-Constraint control. This paper describes the six towers, applying Multi-objective level balance Control System to analysis them.

\section{The process of the Multi-objective Level Balance Control System}

As shown in fig.1, Ore from the underground, by the broken step to the yard for heap leaching, then let the leaching original liquid down to the pool. When open the feed valve, original liquid flow into the first tower for adsorbing, adsorption material adsorb the needed metal ions from the original liquid selectively. When the adsorption material has being saturated, close the feed valve, and open discharge valve to let adsorption material emission to the pressure tank. Then raised the adsorption material to the second tower for cleaning, the third tower, the 4th tower and the 5th tower are for leaching, finally, send the adsorption material into the 6th tower, then raised it to the first tower for adsorbing and achieve the entire process. This is a cycle, the 6th tower played the role of recover the capacity of the adsorption material[4]. 


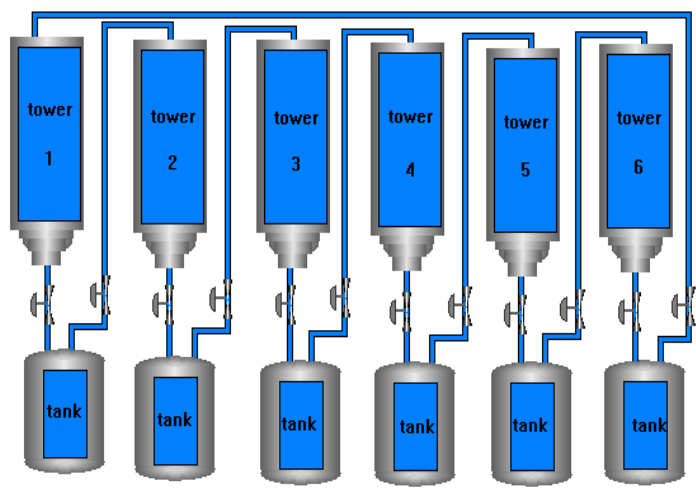

Fig. 1 The process of the Multi-objective Level Balance Control System

During all the production process, a certain amount of adsorption material in every tower needs emission to next tower to deal with when the adsorption material has being saturated. So we should emission from time to time, in other words, we should adjust once in every production cycle. According to the requirement of production process, we must keep the height of tower in the same level, in order to improve the production efficiency.

\section{The Requirement of the Multi-objective Level Balance Control System}

(1) We should adjust the level of six towers once in each production cycle;

(2) There exists a certain range of the adsorption material flows to the next tower, in detail, a maximum of press tank's volume's $90 \%$, a minimum of volume's 50\%;

(3) After the final adjustment, each tower's level and the ideal height's deviation must less than $2 \%$;

(4) The required cycles to achieve balance is the minimum.

\section{Construction of the Multi-objective Level Balance Control System's model}

\section{Explanation of symbols}

$h_{1}(0), h_{2}(0), h_{3}(0), h_{4}(0), h_{5}(0), h_{6}(0)$ : the initial level of six towers;

$h_{v}$ : the ideal level, the average height of the initial level;

$h_{i}^{k}:$ the I-th tower's level after the K-th cycle;

$Q_{m i}^{k}$ : the amount of adsorption material inflow to the I-th tower during the K-th cycle;

$Q_{c i}^{k}$ : the amount of adsorption material outflow from the I-th tower during the K-th cycle;

$V$ : pressure tank's volume;

$s$ : each tower's cross-sectional area;

\section{Construction of model}

In order to ensure the product quality and reduce the cost, it requires each tower reaching the same level after several productive cycles. In this process, each tower's level related to the former tower's adsorption material and next tower's adsorption material. As fig. 1 shown, there are six towers to feed, delay at the same time. The simplified process is shown in fig.3, where we suppose all the adsorption material to be discharged to next tower.

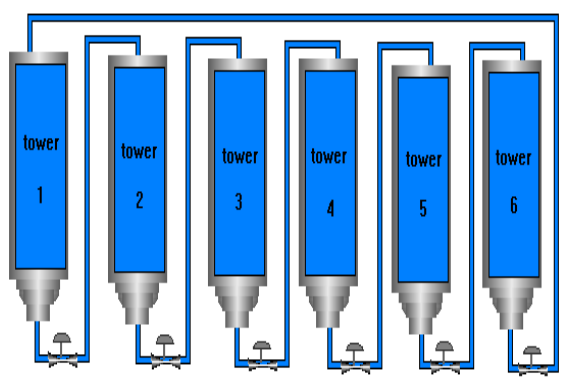

Fig.2 Simplified process 
In industrial production, each tower's shape and size is the same, assuming that the diameter of the tower $\left(R_{1}\right)$ is $1 \mathrm{~m}$, the height $\left(H_{1}\right)$ is $13 \mathrm{~m}$. Then the cross-sectional area of the tower $(\mathrm{s})$ is $0.785 \mathrm{~m} 3$.

This paper is to control the adsorption material's outflow from each tower during each cycle, and make sure each tower reaching the same level finally. Since the six tower to form a loop within the single circulation, then the ideal level $\left(h_{v}\right)$ is the average height of the initial level.

As mentioned in the requirement of the system, after the final adjustment, each tower's level and the ideal height's deviation must less than $2 \%$, in detail,

$$
0.98 h_{v} \leq h_{i} \leq 1.02 h_{v}
$$

From fig.2, it is obvious that the first tower's adsorption material flow into the second tower, the second tower's adsorption material flow into the third tower,....., the 6th tower's adsorption material flow into the first tower, then we have:

$$
\left\{\begin{array}{l}
Q_{m(i+1)}^{k}=Q_{c i}^{k}, i=1, \mathrm{~L} 5 \\
Q_{m(1)}^{k}=Q_{c 6}^{k}
\end{array}\right.
$$

The initial height can described in matrix form, as the same method, the amount of adsorption material outflow from each tower during the K-th cycle and the amount of adsorption material inflow into each tower during the K-th cycle can described in matrix form, as follows:

$$
\begin{aligned}
& h(0)=\left[\begin{array}{llllll}
h_{1}(0) & h_{2}(0) & h_{3}(0) & h_{4}(0) & h_{5}(0) & h_{6}(0)
\end{array}\right]^{\prime}, \\
& Q_{c}^{k}=\left[\begin{array}{llllll}
Q_{c 1}^{k} & Q_{c 2}^{k} & Q_{c 3}^{k} & Q_{c 4}^{k} & Q_{c 5}^{k} & Q_{c 6}^{k}
\end{array}\right], \\
& Q_{m}^{k}=\left[\begin{array}{llllll}
Q_{m 1}^{k} & Q_{m 2}^{k} & Q_{m 3}^{k} & Q_{m 4}^{k} & Q_{m 5}^{k} & Q_{m 6}^{k}
\end{array}\right], \\
& \text { each tower's level after the K-th cycle is } \\
& h^{k}=\left[\begin{array}{llllll}
h_{1}^{k} & h_{2}^{k} & h_{3}^{k} & h_{4}^{k} & h_{5}^{k} & h_{6}^{k}
\end{array}\right]
\end{aligned}
$$

In control system, assuming that the diameter of the tank $(\mathrm{R} 2)$ is $1.2 \mathrm{~m}$, the height $(\mathrm{H} 2)$ is $1.6 \mathrm{~m}$, then it is easy to derive the volume of the tank (v) is $1.80864 \mathrm{~m}^{3}$.

Tank can accommodate a maximum of volume's $90 \%$, a minimum of volume's $50 \%$, then the amount of the adsorption material flow into the next tower is:

$$
0.904 \leq Q_{i}^{k} \leq 1.628
$$

As the amount of adsorption material flow from the former tower to next tower have constraints, then if there are large differences among the six towers initial level, one production cycle can not meet the requirement which is the gap between the ideal level and every tower's level. Only after several production cycles can we meet the requirement.

Using materials balance principle to construction every tower's math model[6]. Take the first tower for example, the initial level is $h_{1}(0)$, assuming the amount of the adsorption material flow from the 6th tower to the first tower is $Q_{m(1)}^{1}$ during the first cycle, the amount of the adsorption material flow from the first tower to the second tower is $Q_{c 1}^{1}$, then the first tower's level meet the following formula:

$$
h_{1}^{1} s=h_{1}(0) s+Q_{m(1)}^{1}-Q_{c 1}^{1}
$$

In fact, $Q_{m(1)}^{1}=Q_{c 6}^{1}$, then (4) can be concluded that

$$
h_{1}^{1} s=h_{1}(0) s+Q_{c 6}^{1}-Q_{c 1}^{1}
$$

Where $s$ is tower's cross-sectional area.

Similarly, other towers meet the following:

$$
h_{i}^{1} s=h_{i}(0) s+Q_{c(i-1)}^{1}-Q_{c i}^{1}
$$

If the above equations solvable, we can meet the requirement in the first cycle, if there is no solution, we must come into the next cycle.

Take the first tower for example, during the second cycle, the initial level is the result level of the first cycle, namely, 


$$
h_{1}^{2} s=h_{1}^{1} s+Q_{c 6}^{2}-Q_{c 1}^{2}
$$

if we need another cycle, the initial level during the third cycle is the result level of the second cycle, namely,

$$
h_{1}^{3} s=h_{1}^{2} s+Q_{c 6}^{3}-Q_{c 1}^{3}
$$

likewise, if we need the K-th cycle, the initial level is the result level of the (K-1)th cycle, in this case,

$$
\begin{gathered}
h_{1}^{k} s=h_{1}^{k-1} s+Q_{c 6}^{k}-Q_{c 1}^{k} \\
h_{1}^{1} \text { with } h_{1}(0), h_{1}^{2} \text { with } \begin{array}{c}
h_{1}^{1}, \ldots, h_{1}^{k} \text { with } h_{1}^{k-1} \text {, then (9) can be written as follows: } \\
h_{1}^{k} s=h_{1}(0) s+Q_{c 6}^{1}+Q_{c 6}^{2}+\mathrm{L}+Q_{c 6}^{k} \\
-Q_{c 1}^{1}-Q_{c 1}^{2}-\mathrm{L}-Q_{c 1}^{k}
\end{array}
\end{gathered}
$$

Similarly, others tower's relationship is

$$
\begin{aligned}
h_{i}^{k} s= & h_{i}(0) s+Q_{c(i-1)}^{1}+Q_{c(i-1)}^{2}+\mathrm{L}+Q_{c(i-1)}^{k} \\
& -Q_{c i}^{1}-Q_{c i}^{2}-\mathrm{L}-Q_{c i}^{k}
\end{aligned}
$$

For the known height of the initial level, if the adjustment cycle the less, the less time required, the sooner the balance, the higher the production efficiency, the higher the economic performance. Therefore, the objective function is the minimum cycle, which is min $\mathrm{k}$.

In summary, the Multi-objective Level Balance Control System's model is

$$
\left\{\begin{array}{c}
h^{k} s=h(0) s+\left[\begin{array}{llll}
Q_{m}^{1} & Q_{m}^{2} & \mathrm{~L} & Q_{m}^{k}
\end{array}\right] \cdot\left[\begin{array}{llllll}
1 & 1 & 1 & 1 & 1 & 1
\end{array}\right] \\
\quad-\left[\begin{array}{llllll}
Q_{c}^{1} & Q_{c}^{2} & \mathrm{~L} & Q_{c}^{k-1} & Q_{c}^{k}
\end{array}\right] \cdot\left[\begin{array}{llllll}
1 & 1 & 1 & 1 & 1 & 1
\end{array}\right] \\
0.904 \leq Q_{i} \leq 1.628, i=1, \mathrm{~L} 6 \\
0.98 h_{v} \leq h_{i}^{1} \leq 1.02 h_{v}
\end{array}\right.
$$

The objective function is $\min \mathrm{k}$.

\section{MATLAB solving and data analysis}

Based on the above model, use matlab to program and solve. In the MATLAB optimization toolbox, there is fmincon function for solving nonlinear programming. Use it like this:

[x, fval, exitflag, output]=fmincon (@myfun, x0, A, b, Aeq, beq, lb, ub, @ nonlcon, options)

Where, myfun is the objective function name, $\mathrm{x} 0$ is the initial of the variable, nonlcon is the non-linear constraint's function name.

In this model, The objective function ( $\min \mathrm{k}$ ) written into myfun, initial level written into $\mathrm{x} 0$, the contents of nonlcon is range of adjustment $\left({ }^{0.904} \leq Q_{i}^{1} \leq 1.628, i=1, \mathrm{~L} 6\right.$ ), equations which made by the material balance principle and the range of error( $\left.0.98 h_{v} \leq h_{i}^{1} \leq 1.02 h\right)$.

In this paper, we use fmincon function to get the best solution, if the level has the large difference, we have to go through several production cycles in order to achieve balance. Assuming that the initial level of the six tower is $11.5 \mathrm{~m}, 7.0 \mathrm{~m}, 7.8 \mathrm{~m}, 9.4 \mathrm{~m}, 8.6 \mathrm{~m}, 10.6 \mathrm{~m}$, the result is $\mathrm{k}=4$, which means needed 4 cycles to achieve balance, and the amount of adsorption material flow in every cycle is as follows:

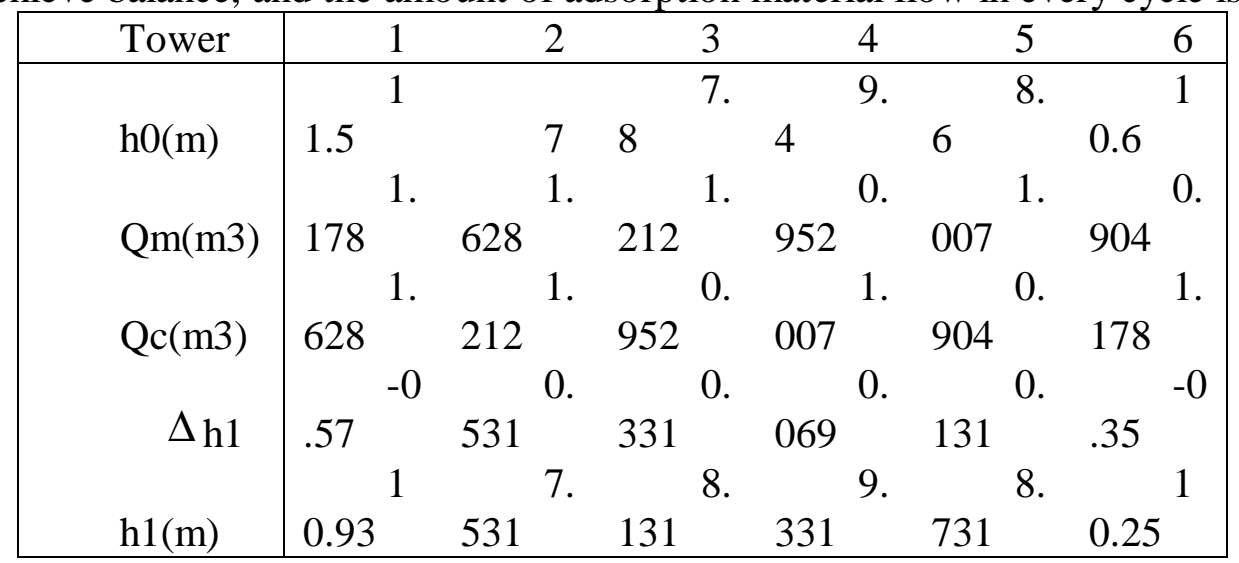

Tab 1 The first cycle's data 
Similarly, during the second cycle, the initial level is the result level of the first cycle, the initial level during the third cycle is the result level of the second cycle, the initial level during the 4th cycle is the result level of the third cycle. So, we obtained the level of every tower and the amount of adsorption material flow to next tower which are given in table 2.

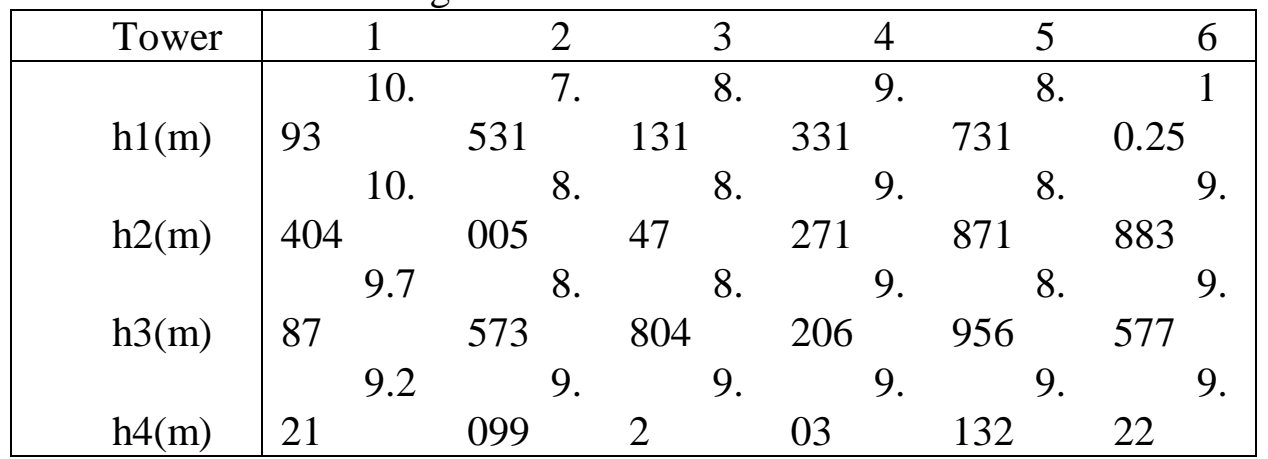

Tab 2 Level after each cycle

Where, Qc is the amount of adsorption material outflow from each tower, Qm is the amount of adsorption material inflow into next tower, $\Delta h_{n}$ is six tower's net increase amount during every cycle, which is

$$
\Delta h_{n}=\left(Q_{m}^{n}-Q_{c}^{n}\right) / s
$$

$h(0)$ is the initial level of each tower, ${ }^{h}$ is the level of six towers after the first cycle, ${ }_{2}$ is the level of six towers after the second cycle, ${ }^{h_{3}}$ is the level of six towers after the third cycle, $h_{4}$ is the level of six towers after the 4 th cycle.

Compared to the level of six towers after every cycle, the level tends to the ideal level, which is given in fig. 3 .

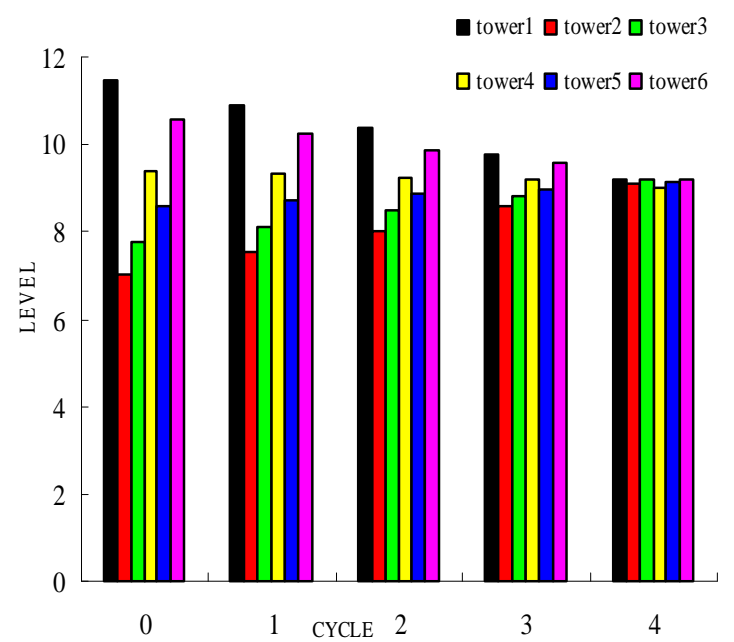

Fig.3 After every cycle's level of six tower

As illustrated in Fig. 3, the cycle 0 is the initial level, cycle2, cycle 3, cycle 4 is after the second cycle, the third cycle, the fourth cycle's level of six towers, respectively, it is obvious known that after each cycle the level of six towers are gradually moving to the average level, then proved the validity of the model.

During the process, the amount of adsorption material inflow and outflow from the six towers are not the same in each cycle, it is given in fig.4 


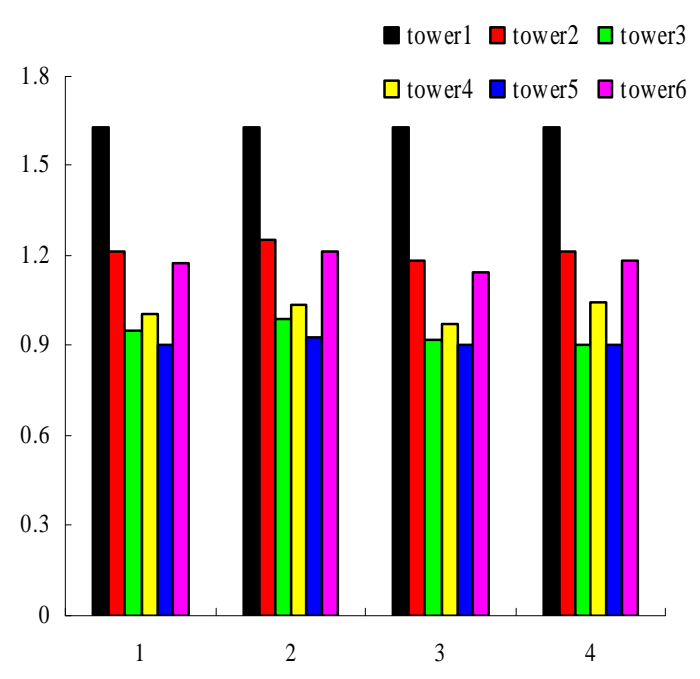

Fig.4 The amount of adsorption material

Since the first tower's initial level is relatively large, so that the amount of the adsorption material outflow was relatively large in each cycle, others smaller. It is clearly seen each cycle's amount of the adsorption material outflows from every tower.

Analysis of the data in table 3, the relative error of the final level and the average of the initial level are shown in tab 3.

\begin{tabular}{|c|cccccc|}
\hline tower & 1 & 2 & 3 & 4 & 5 & 6 \\
\hline deviatio & 0.8 & 0.6 & 0.5 & 1.3 & 0.2 & 0.8 \\
$\mathrm{n}$ & $\%$ & $\%$ & $\%$ & $\%$ & $\%$ & $\%$ \\
\hline
\end{tabular}

Tab3 Deviation of each tower

As shown in Tab 3, the final level and the average of the initial level's relative error are less than $2 \%$, so the solution can meet the requirements. In this system, collected the liquid level sensor's result, and compared with adjustable value, when the level trends to convergence, during the next cycle, the adjustable value is the maximum $(1.628 \mathrm{~m} 3)$. So that the level will keeping balance and reaching the optimal productivity. For any given initial value, this model can calculate the minimum cycle and the amount of outflow from each tower during every cycle, proved the effectiveness and feasibility of the model.

\section{Conclusion}

In this paper, we have established a model of Multi-objective Level Balance Control System by material balance principle. Programmed and solved the model by matlab, the results show that the mathematical model can apply to keep the level of six towers within the same height, which has advantages such as adjust the level automatically, high adsorption efficiency, low cost and so on. Thus the competitiveness of enterprises would be highly improved.

\section{References}

[1] Gavin M.Mudd. Acid in Situ Leach Uranium Mining-USA an Australia [J]. Tailings \& Mine Waste. January: pp.23-26, (2000).

[2] Uranium Energy Corporation. Palangana Hobson Operation Tour [J]. Vancouver: Uranium Energy Corporation, (2010).

[3] Michael B.Mooiman. Challenging the traditional hydrometallurgy curriculum —an industry perspective [J]. Hydrometallurgy, 79, pp.80- 88 (2005).

[4] The extraction of adsorption material in the metal and other useful components [J]. Beijing: Metallurgical Industry Press, pp.47-72,(1995).

[5] Zhao Tinggang. Mathematical modeling methods and mathematical models [M]. Beijing: Science Press, (2011). 
[6] Wang Xiaopeng. Three-Tank water tank level control system dynamic simulation and multi-line monitoring of process variables [D]. Shandong University master's degree thesis .2005.05.

[7] Simon Hecker. Enhanced LFR-toolbox for Matlab[J]. Aerospace Science and Technology 9, pp.173-180, (2005). 